\title{
Sensitivity Analysis of the Simply Noise-matched Receiving Coil for NMR Experiments
}

\author{
Peter Andris, Ivan Frollo \\ Institute of Measurement Science, Slovak Academy of Sciences, Dubravska cesta 9, 841 04, Bratislava, Slovak republic, \\ peter.andris@savba.sk
}

\begin{abstract}
The article analyzes the sensitivity of unmatched receiving coil for the NMR scanner. Receiver of the scanner was investigated from the point of view of noise features. Theory of the noise figure has been modified to utilize the receiver for digitization of its own noise and the noise figure calculation. The resulting noise figure has been measured with different source impedances and the optimal value has been acquired. Influence of the noise figure on the resulting signal-to-noise ratio has been calculated for the sensitivity judgement. The output SNR has been investigated for constant input SNR as well as for constant input voltage. Many results are depicted in figures. Also examples of theoretical results are depicted graphically.
\end{abstract}

Keywords: NMR, scanner, receiver, noise figure, SNR, noise matching.

\section{INTRODUCTION}

Electronic parts operating at higher frequencies using transmission lines are matched to a defined characteristic impedance of a transmission line. Input and output impedances of the electronic parts must be matched to that impedance for preventing distortions and losses of signals. Thus, the circuits are matched from the point of view of impedance and from the point of view of the power transfer simultaneously. Sensitivity of an electronic part depends not only on the gain but also on noise features and this is important [1]-[8]. Noise qualities of an electronic circuit can be described by its noise figure. Theory of the noise figure shows that the noise figure versus the source impedance is a function with a minimum. If the input of the receiver is connected to the optimal source impedance, the gain of the receiver may not be maximal, but its noise figure is minimal. It can be said that the receiver is matched from the point of view of noise. The purpose of the research is to utilize it for a receiving coil of the NMR scanner with maximal gain and sensitivity. A novel technique of the noise figure measurement had been developed [3] and it was partly used for the noise optimum of the NMR scanner receiver search [4]. The technique does not require any special instruments, the NMR receiver can be utilized for the noise measurement. The measured quantities can be processed using a standard $\mathrm{PC}$. The relation between the noise figure of the receiver and the signal-to-noise ratio (SNR) at the output of the receiver is a suitable tool for sensitivity judgement. Generally, the source impedance is a complex number. The minimal noise figure is a function of the source impedance module as well as of its phase [2]. The calculations in this article are approximative, only source resistances are considered and only on the resonant frequencies. Moreover, the complex numbers would require more complex and more expensive instruments that are not available in most NMR laboratories.

References [9]-[19] solve interesting problems, similar to the problems solved in this article. The processing of the measured data is discussed elsewhere [20]. The theory and results described in the present article are useful for technical and experimental purposes. They can provide a platform for following theoretical and experimental studies. Our research offers options for techniques which utilize the NMR spectroscopic console, a frequent equipment of many NMR laboratories. The technique has been tested at the apparatus for measurements at low magnetic field but there is no reason why it could not be used also for higher spectra frequencies. The results of the research are partly experimental and partly theoretical.

\section{SUBJECT \& METHODS}

Fig.1. depicts an equivalent circuit diagram of an NMR receiver. For simplicity, all impedances are considered as resistances. It corresponds well to reality, for the value of the transmission line characteristic impedance is a real number. At the operation frequency, impedance of the signal source is a real number (resonance). Signal source is represented by its resistance $R_{g}$ and voltage $V_{g}$. In practice it is a receiving coil with its tuning and matching circuitry. Sources of noise are 
all resistances in this calculation. Influence of noise can be described by the signal-to-noise ratio $(S N R) . S N R_{1}$ is defined at the input of the receiver, $S N R_{2}$ in the load resistance of the receiver output. $S N R_{1}$ is given by (1):

$$
S N R_{1}=\frac{\frac{V_{1_{S}}^{2}}{R_{i n}}}{\frac{4 k T R_{g} \Delta f}{\left(R_{g}+R_{i n}\right)^{2}} \cdot R_{i n}}=\frac{V_{1_{S}}^{2}\left(R_{g}+R_{i n}\right)^{2}}{R_{i n}^{2} 4 k T R_{g} \Delta f}
$$

$$
\begin{array}{cl}
\text { where } & \\
V_{1_{s}} & \text { rms signal voltage at the input of the receiver } \\
R_{g} & \text { output resistance of the signal source (receiving coil) } \\
R_{\text {in }} & \text { input resistance of the receiver } \\
k & \text { the Boltzmann constant } \\
T & \text { absolute temperature of all resistances } \\
\Delta f & \text { noise bandwidth of the measuring instrument } \\
R_{2} & \text { load resistance in the output of the receiver }
\end{array}
$$

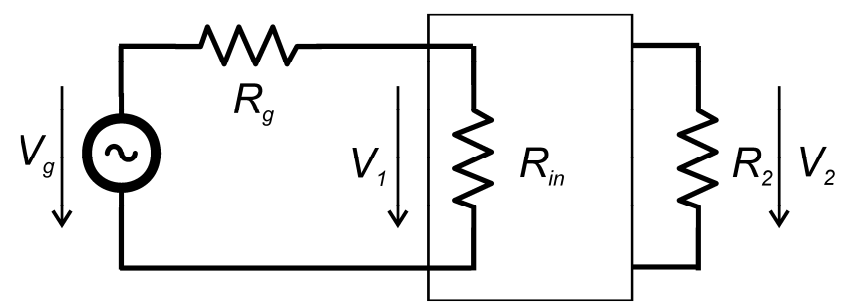

Fig.1. Equivalent circuit diagram of the NMR receiver. Impedances have their real values at the operation frequency.

Generally, $R_{g} \neq R_{\text {in }}$. Equation (2) shows the noise power produced by $R_{g}$ and transferred into the receiver input:

$$
P_{1_{n}}=\frac{4 k T R_{g} \Delta f}{\left(R_{g}+R_{i n}\right)^{2}} \cdot R_{i n}
$$

The situation is depicted in the equivalent circuit diagram in Fig.2.

$S N R_{2}$ is given by (3):

$$
S N R_{2}=\frac{V_{2_{S}}^{2}}{V_{2_{n}}^{2}}
$$

where

$V_{2_{s}} \quad$ rms signal voltage at the load resistor $R_{2}$

$V_{2_{n}} \quad$ rms noise voltage at the load resistor $R_{2}$

Both voltages are assumed to be in $\mathrm{A} / \mathrm{D}$ converter units.

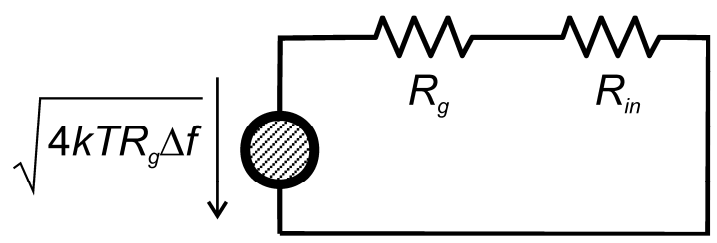

Fig.2. Equivalent circuit diagram of the NMR receiver from the point of view of noise.
Noise factor of the receiver can be defined in more ways. A frequent definition uses a ratio of input and output $S N R$ : (4).

$$
F=\frac{S N R_{1}}{S N R_{2}}=\frac{V_{1_{s}}^{2}}{V_{2_{S}}^{2}} \cdot \frac{\left(R_{g}+R_{i n}\right)^{2} \cdot V_{2 n}^{2}}{R_{i n}^{2} \cdot 4 k T R_{g} \Delta f}=K^{2} \cdot \frac{\left(R_{g}+R_{i n}\right)^{2} \cdot V_{2_{n}}^{2}}{R_{i n}^{2} \cdot 4 k T R_{g} \Delta f}
$$

The calibration coefficient $K$ can be measured using a suitable signal generator and the console of the NMR receiver. Whereas $F$ should be unitless, also $V_{2_{s}}$ should be in $\mathrm{A} / \mathrm{D}$ converter units. If using a generator of harmonic signal for the $K$ calculation, the RMS voltage in $K$ may be replaced by its amplitude or mean value. The noise figure in decibels is given by (5):

$$
N F=10 \cdot \log _{10} F
$$

After measurement, the voltages $V_{2}$ have the form of time sample series. To determine the noise bandwidth properly, they must be converted into a series of frequency samples using the discrete Fourier transform. Consider that $V$ is given by $N$ frequency samples. RMS value of the whole series is given by (6):

$$
V_{2_{\mathrm{n} r m s}}=\sqrt{\frac{1}{N^{2}} \sum_{i=1}^{N}\left|V_{i}\right|^{2}}=\sqrt{\sum_{i=1}^{N}\left|\frac{V_{i}}{N}\right|^{2}}
$$

In practice, only such interval of the samples should be selected, so that the spectrum within the interval could be considered constant. For cropping of noise bandwidth, (6) can be modified to (7):

$$
V_{2_{n_{r m s}}}=\sqrt{\left(\frac{1}{N}\right)^{2} \cdot \sum_{i=n_{1}}^{n_{2}}\left|V_{i}\right|^{2}}
$$

where $1 \leq n_{1}<n_{2} \leq N$.

The resulting equation for the noise factor is given by (8):

$$
F=K^{2} \cdot \frac{\left(R_{g}+R_{i n}\right)^{2} \cdot\left(\frac{1}{N}\right)^{2} \cdot \sum_{i=1}^{d}\left|V_{i}\right|^{2}}{R_{i n}^{2} \cdot 4 k T R_{g} \Delta f}
$$

where

d number of selected frequency samples.

\section{RESULTS}

Verification experiments were performed on an experimental NMR scanner equipped with a home-made resistive magnet of $0.1 \mathrm{~T}$ and the Apollo spectroscopic NMR console (Tecmag Inc., Houston, TX). The console was utilized for imaging, operated at a frequency of $4.45 \mathrm{MHz}$. Console calibration was performed using the GFG-3015 function generator (Good Will Instrument Co., Ltd., New Taipei City, Taiwan).

An AU-1579 preamplifier (MITEQ, Hauppauge, NY) with $50 \Omega$ input and output impedances and $N F=1 \mathrm{~dB}$ (a catalogue datum) was used. The preamplifier is not intended for operations in magnetic field. 


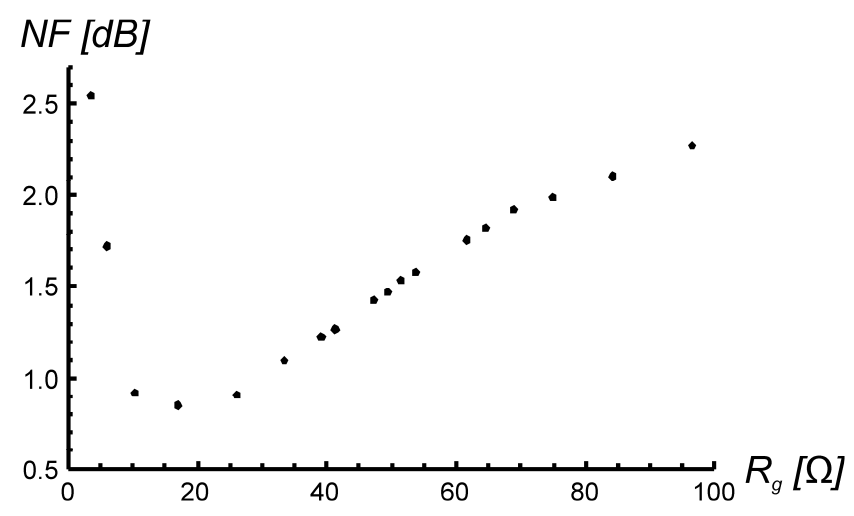

Fig.3. The noise figure depending on the source resistance $R_{g}$ measured with the above described technique.

The noise figure of the NMR receiver has been measured with altering source resistance using a variable attenuator. The results are depicted in Fig.3. for $N=500000$ and $d=300000$.

Modifying (4) $S N R_{2}$ can be calculated (9):

$$
S N R_{2}=\frac{\frac{S N R_{1}}{F}}{\operatorname{Part}\left[\frac{S N R_{1}}{F}, R_{g}=50 \Omega\right]}
$$

Considering $S N R_{1}=$ const. are calculated data in Fig.4., the values are normed to the $S N R_{2}$ corresponding to $R_{g}=50 \Omega$.

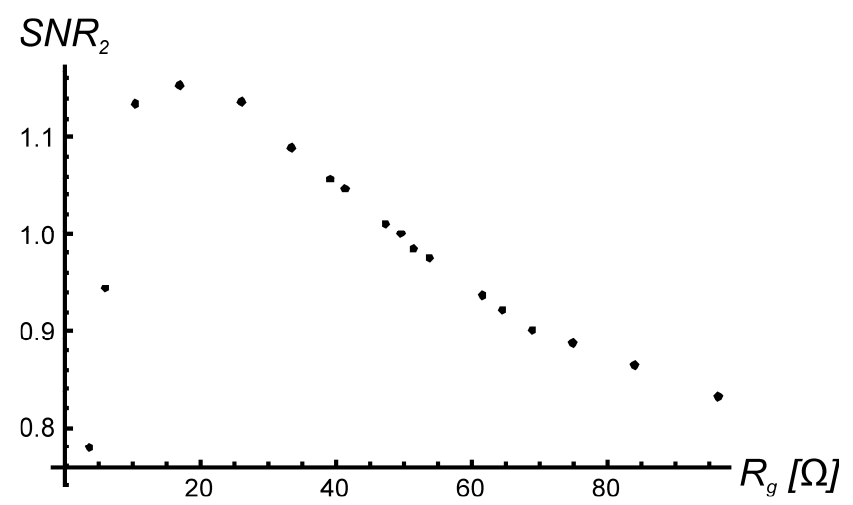

Fig.4. $S N R_{2}$ at the output of the receiver considering $S N R_{1}=$ const. The values are normed to the value corresponding to $R_{g}=50 \Omega$.

A constant $S N R_{1}$ is not frequent in practice. Consider constant $V_{g}$, the RMS value of the harmonic input signal voltage. The coil is connected to the receiver via a known tuning and matching circuit, but it is not matching the coil to input resistance of the receiver $R_{\text {in }}$. The circuit is depicted in Fig.5. The coil is tuned and matched to resistances $R_{g}$, nevertheless it is connected to the resistance $R_{\text {in }}$. For our example the value $V_{g}=10^{-7} \mathrm{~V}$ has been adopted. Other quantities:

$f_{0}=4.45 \mathrm{MHz}$

$Q_{c}=250$ is quality factor of the unloaded coil

$X_{c}=\omega_{0} L_{c}=75 \Omega$ is reactance of the coil
$R_{\text {out }}$ is output resistance of the tuning and matching circuit, corresponding to $R_{g}$

$R_{c}=\frac{\omega_{0} L_{c}}{Q_{c}}$ is resistance of the unloaded coil

Values of the capacitances $C_{1}$ and $C_{2}$ are given by (10) and (11):

$$
\begin{gathered}
C_{1}=\frac{-R_{c}^{2} \sqrt{R_{C}}+R_{C} R_{g} \sqrt{R_{C}}-X_{c}^{2} \sqrt{R_{c}}+X_{c} \sqrt{R_{g}\left(R_{c}^{2}-R_{c} R_{g}+X_{c}^{2}\right)}}{\omega_{0}\left(R_{c}^{2}+X_{c}^{2}\right) \sqrt{R_{g}\left(R_{c}^{2}-R_{C} R_{g}+X_{c}^{2}\right)}} \\
C_{2}=\frac{1}{\omega_{0}} \sqrt{\frac{R_{C}}{\sqrt{R_{g}\left(R_{C}^{2}-R_{c} R_{g}+X_{C}^{2}\right)}}}
\end{gathered}
$$

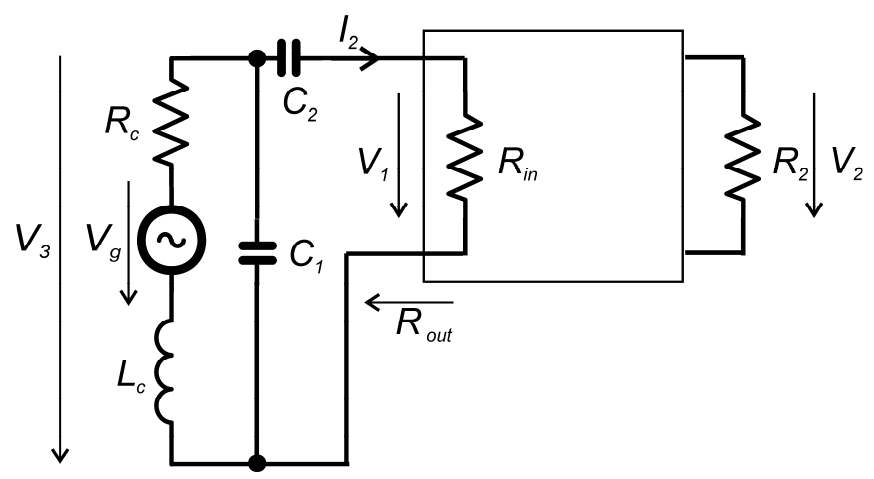

Fig.5. Example with constant $V_{g}$ voltage. The coil is connected to the receiver through the capacitive tuning and matching circuit. Resistance $R_{\text {out }}$ is the source of the thermic noise. It replaces $R_{g}$ from the first example.

Auxiliary voltage $V_{3}$ is given by (12):

$$
\begin{aligned}
V_{3} & =\frac{V_{g}}{R_{c}+\mathbf{j} \omega_{0} L_{c}} \cdot \frac{1}{\frac{1}{R_{c}+\mathbf{j} \omega_{0} L_{c}}+\mathbf{j} \omega_{0} C_{1}+\frac{1}{R_{\text {in }}+\frac{1}{\mathbf{j} \omega_{0} C_{2}}}}= \\
& =\frac{V_{g}}{\left(\mathbf{j} \omega_{0} C_{1}+\frac{\omega_{0} C_{2}}{\mathbf{j} \omega_{0} C_{2} R_{\text {in }}}+\frac{1}{R_{C}+\mathbf{j} X_{C}}\right)\left(R_{C}+\mathbf{j} X_{C}\right)}
\end{aligned}
$$

Auxiliary current $I_{2}$ is given by (13):

$$
\begin{gathered}
I_{2}=\frac{V_{3}}{\frac{1}{\mathbf{j} \omega C_{2}}+R_{\text {in }}}= \\
=\frac{-\mathbf{j} \omega_{0} C_{2} V_{g}}{-1+\omega_{0}\left(C_{1}\left(-\mathbf{j}+\omega_{0} C_{2} R_{i n}\right)\left(R_{C}+\mathbf{j} X_{C}\right)+C_{2}\left(-\mathbf{j}\left(R_{C}+R_{i n}\right)+X_{C}\right)\right)}
\end{gathered}
$$

The signal power in-coming into the receiver is given by (14):

$$
P_{i n_{S}}=\operatorname{Re}\left[I_{2}^{2} R_{\text {in }}\right]
$$

The signal power versus $R_{g}$ is depicted in Fig.6. 
The voltage $V_{1_{s}}$ in the input of the receiver is given by (15):

$$
V_{1_{s}}=\operatorname{Re}\left[I_{2} R_{\text {in }}\right]
$$

The signal voltage is depicted in Fig. 7.

Output resistance of the capacitive circuit is given by (16):

$$
R_{o u t}=\frac{R_{c}}{\omega^{2} R_{c}^{2} C_{1}^{2}+\left(\omega^{2} L_{c} C_{1}-1\right)^{2}}
$$

Output resistance of the tuning and matching circuit versus frequency is depicted in Fig.8. Source resistance $R_{g}=50 \Omega$. Noise bandwidth $\Delta f=\frac{d}{N T_{s}}, T_{s}$ is sampling interval of the A/D converter.

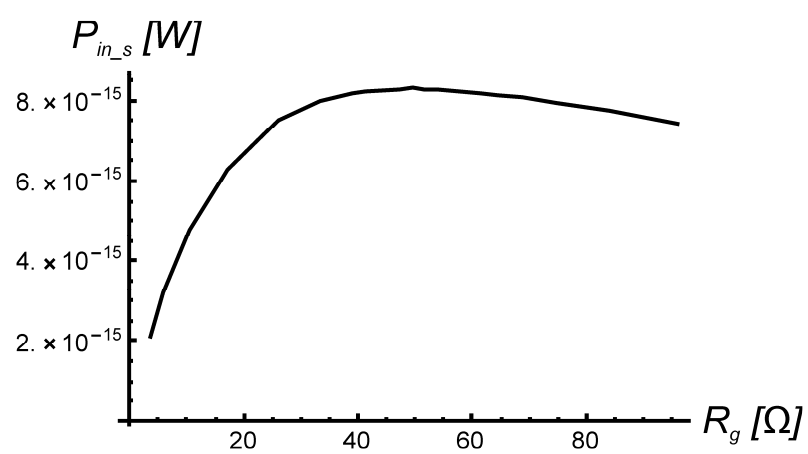

Fig.6. The signal power in-coming into the receiver.

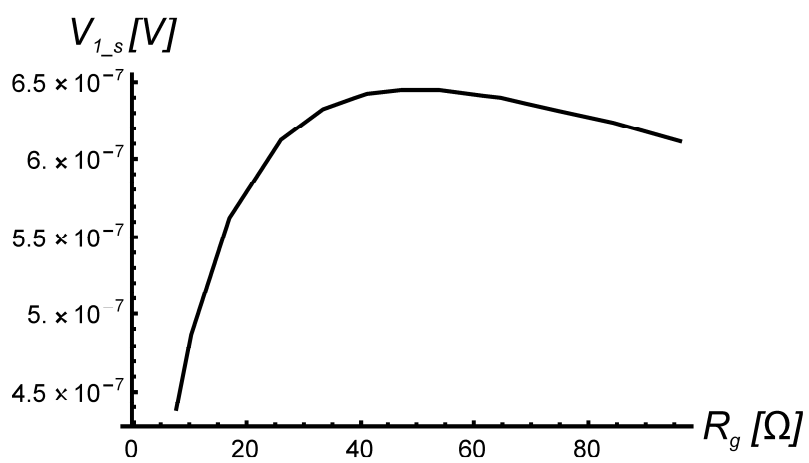

Fig.7. The signal voltage at the input of the receiver versus $R_{g}$.

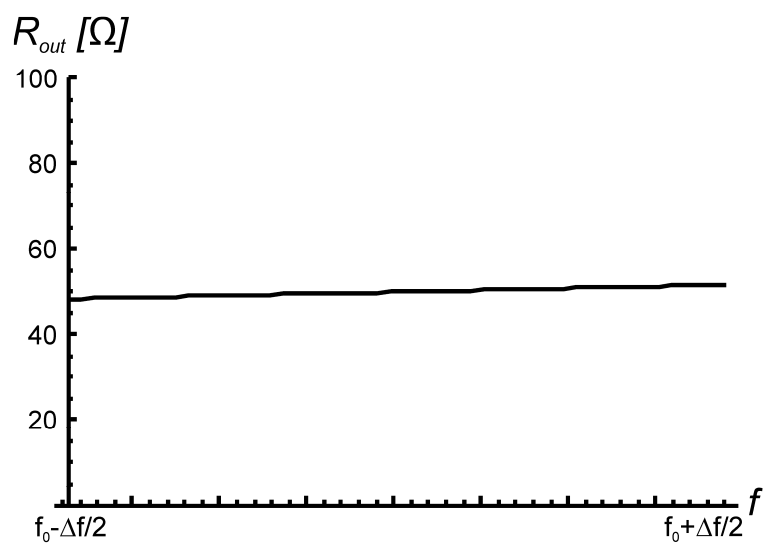

Fig.8. Real part of the tuning and matching circuit output impedance versus frequency. $R_{g}=50 \Omega$.
$S N R_{1}$ in the input of the receiver is given by (17), depicted in Fig.9.

$$
S N R_{1}=\frac{\left(\bar{R}_{\text {out }}+R_{\text {in }}\right)^{2}}{R_{\text {in }}} \cdot \frac{P_{\text {in }}}{\int_{f_{0}-\frac{\Delta f}{2}}^{f_{0}+\frac{\Delta f}{2}} 4 k T R_{\text {out }} d f} \cong \frac{V_{1_{S}}^{2}\left(R_{g}+R_{\text {in }}\right)^{2}}{R_{\text {in }}^{2} 4 k T R_{g} \Delta f}
$$

It is obvious that values of $S N R_{1}$ are very close to a constant. $S N R_{2}$ is calculated using (9) again, therefore values and shape are also extremely close to that in the first example. $S R_{2}$ is depicted in Fig.10.

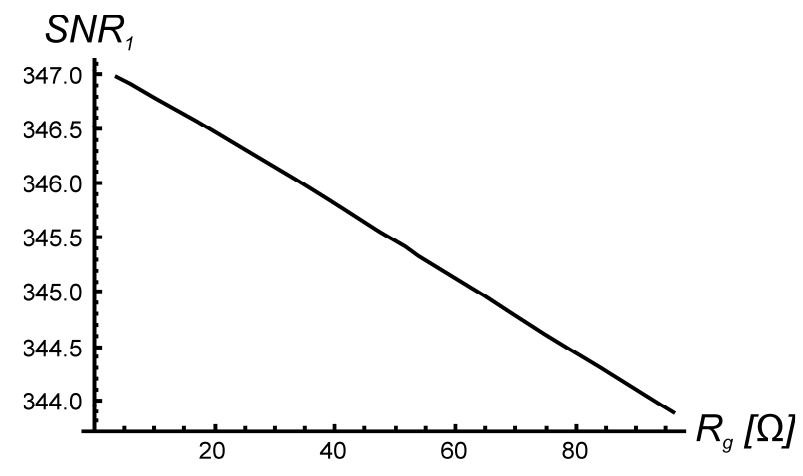

Fig.9. $S N R_{1}$ versus the source resistance.

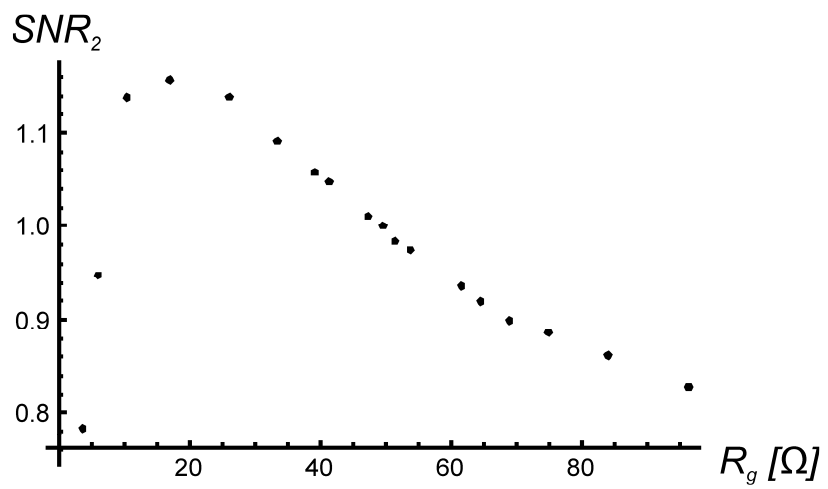

Fig.10. The values and shape of $S N R_{2}$ with $V_{g}=$ const. are very close to that from the first example with $S N R_{1}=$ const. (Fig.4).

\section{DISCUSSION / CONCLUSIONS}

The purpose of this study was to look for minimal noise in the NMR experiment with simple tools. The noise matching is not absolutely accurate but sufficient for the practice and can be an introduction for more advanced research. The noise matched receiving coil provides better sensitivity, compared to 50 -Ohm matching. However, the connection between the coil and the receiver must be carried out more accurately, it must be as short as possible, some parts of the receiver must be able to operate in the static magnetic field of the NMR scanner. Significant part of the receiver is the preamplifier, it influences noise features of the receiver strongly. It is the receiver part operating in the static magnetic field. Standard matched coils are usually matched to $50 \Omega$ load. Such coil can be also noise-matched but it may not be. The described technique is suitable for preparation of special experiments with high requirements on sensitivity. 


\section{ACKNOWLEDGMENT}

This work was supported by the Scientific Grant Agency VEGA 2/0003/20, and within the project of the Research and Development Agency No. APVV-19-0032.

\section{REFERENCES}

[1] Žalud, V., Kulešov, V.N. (1980). Polovodičové obvody $s$ malým šumem (Semiconductor Circuits with Low Noise). Prague, Czech Republic: SNTL. (in Czech)

[2] Schiek, B., Siweris, H.J. (1990). Rauschen in Hochfrequenz-schaltungen (Noises in RF Circuits). Heidelberg, Germany: Hüthig. (in German)

[3] Andris, P., Emery, E.F., Frollo, I. (2019). Analysis of NMR spectrometer receiver noise figure. Mathematical Problems in Engineering, 2019, 1083706. https://doi.org/10.1155/2019/1083706.

[4] Andris, P., Dermek, T., Frollo, I. (2019). Noise matching of the NMR scanner receiver. In MEASUREMENT 2019: 12th International Conference on Measurement. Bratislava, Slovakia: Institute of Measurement Science, Slovak Academy of Sciences, 274-277. https://doi.org/10.23919/MEASUREMENT 47340.2019.8779891.

[5] Hoult, D.I., Richards, R.E. (1976). The signal-to-noise ratio of the nuclear magnetic resonance experiment. Journal of Magnetic Resonance 24 (1), 71-85. https://doi.org/10.1016/0022-2364(76)90233-X.

[6] Hoult, D.I., Lauterbur, P.C. (1979). The sensitivity of the zeugmatographic experiment involving human samples. Journal of Magnetic Resonance, 34 (2), 425433. https://doi.org/10.1016/0022-2364(79)90019-2.

[7] Raad, A., Darrasse, L. (1992). Optimization of NMR bandwidth by inductive coupling. Magnetic Resonance Imaging, 10 (1), 55-65. https://doi.org/10.1016/0730725x(92)90373-8.

[8] Décorps, M., Blondet, P., Reutenauer, H., Albrand, J.P., Remy, C. (1985). An inductively coupled, series-tuned NMR probe. Journal of Magnetic Resonance, 65 (1), 100-109. https://doi.org/10.1016/0022-2364(85)903786.

[9] Andris, P. (2001) Matching and tuning RF coils for NMR tomograph. Measurement Science Review, 1 (1), 115-118.

[10] Andris, P., Frollo, I. (2003). Matching of RF coils for NMR measurements using inductors. Measurement Science Review, 3 (3), 57-60.

[11] Vergara Gomez, T.S., Dubois, M., Glybovski, S., Larrat, B., de Rosny, J., Rockstuhl, C., Bernard, M., Abdeddaim, R., Enoch, S., Kober, F. (2019). Wireless coils based on resonant and nonresonant coupled- wire structure for small animal multinuclear imaging. $N M R$ in Biomedicine, 32 (5), e4079. https://doi.org/10.1002/ nbm.4079.
[12] Qian, Ch., Duan, Q., Dodd, S., Koretsky, A., MurphyBoesch, J. (2016). Sensitivity enhancement of an inductively coupled local detector using a HEMT-based current amplifier. Magnetic Resonance in Medicine, 75 (6), 2573-2578. https://doi.org/10.1002/mrm.25850.

[13] Weis, J., Ericsson, A., Hemmingsson, A. (1999). Chemical shift artifact-free microscopy: Spectroscopic microimaging of the human skin. Magnetic Resonance in Medicine, 41 (5), 904-908. https://doi.org/10.1002/ (SICI)1522-2594(199905)41:5\%3C904::AID-MRM8 \%3E3.0.CO;2-4.

[14] Marcon, P., Bartusek, K., Dokoupil, Z., Gescheidtova, E. (2012). Diffusion MRI: Mitigation of magnetic field inhomogeneities. Measurement Science Review, 12 (5), 205-212. https://doi.org/10.2478/v10048-012-0031-8.

[15] Bartusek, K., Dokoupil, Z., Gescheidtova, E. (2007). Mapping of magnetic field around small coil using the magnetic resonance method. Measurement Science and Technology, 18 (7), 2223-2230. https://doi.org/ 10.1088/0957-0233/18/7/056.

[16] Nespor, D., Bartusek, K., Dokoupil, Z. (2014). Comparing saddle, slotted-tube and parallel-plate coils for Magnetic Resonance Imaging. Measurement Science Review, 14 (3), 171-176. https://doi.org/ 10.2478/msr-2014-0023.

[17] Latta, P., Gruwel, M.L., Volotovskyy, V., Weber, M.H., Tomanek, B. (2007). Simple phase method for measurement of magnetic field gradient waveforms. Magnetic Resonance Imaging, 25 (9), 1272-1276. https://doi.org/10.1016/j.mri.2007.02.002.

[18] Latta, P., Gruwel, M.L., Volotovskyy, V., Weber, M.H., Tomanek, B. (2008). Single-point imaging with a variable phase encoding interval. Magnetic Resonance Imaging, 26 (1), 109-116. https://doi.org/10.1016/ j.mri.2007.05.004.

[19] Gupta, M., Safvan, C.P., Singh, K., Lobiyal, D.K. (2018). Modeling and simulation of on-chip probe for portable NMR applications. In Progress in Electromagnetics Research Symposium (PIERSToyama). IEEE, 1918-1924. https://doi.org/10.23919/ PIERS.2018.8597770.

[20] Wimmer, G., Witkovský, V., Duby, T. (2000). Proper rounding of the measurement results under normality assumptions. Measurement Science and Technology, 11 (12), 1659-1665. https://doi.org/10.1088/0957$0233 / 11 / 12 / 302$.
Received May 20, 2020

Accepted October 5, 2020 